\title{
Mulheres e álcool: padrão de consumo e fatores associados
}

\section{Women and alcohol: consumption standard and associated factors}

\author{
Eukália Pereira da Rocha' • Claudete Ferreira de Souza Monteiro ${ }^{\bullet}$ Jaqueline Carvalho e Silva Sales $^{3}$ \\ Lorena Uchoa Portela Veloso ${ }^{4}$ Fernando José Guedes da Silva Junior ${ }^{5} \bullet$ Thiago Alberto de Souza Monteiro $^{6}$
}

\begin{abstract}
RESUMO
Objetivo:Analisar o padrão de consumo do álcool e fatores associados em mulheres. Método: Estudo transversal, com 305 mulheres na faixa de 20 a 59 anos, atendidas em unidades básicas de saúde em Teresina/Piauí/Brasil. Utilizou-se Escala Alcohol Use Desorders Identification Test para identificar o padrão de consumo do álcool e questionário para variáveis sociodemográficas, de saúde e hábitos de vida, com coleta de maio a junho de 2019 e analisados pelo software Statistical Package for the Social Science, versão 20.0. Resultados: A prevalência de álcool foi 34,4\%, índice de binge drink de $63,8 \%$. O padrão de consumo mostrou que $84,9 \%$ apresentaram baixo risco/ abstinentes, II, $1 \%$ uso de risco, I,3\% uso nocivo e 2,6\% possível dependência. Houve associação entre o consumo de álcool com escolaridade, violência e discriminação. Conclusão: Alta prevalência associada a fatores como violência, discriminação e escolaridade, requer maior atenção às mulheres usuárias de álcool.
\end{abstract}

Palavras-chave: Mulheres;Abuso de álcool;Atenção primária à saúde; Fatores de risco.

\begin{abstract}
Objective:To analyze the pattern of alcohol consumption and associated factors in women.

Method: Cross-sectional study, with 305 women aged 20 to 59 years, attended at basic health units in Teresina / Piauí / Brazil.The Alcohol Use Desorders Identification Test Scale was used to identify the pattern of alcohol consumption and a questionnaire for sociodemographic, health and lifestyle variables, collected from May to June 2019 and analyzed using the Statistical Package for the Social Science software, version 20.0. Results: The prevalence of alcohol was 34.4\%, with a binge drink rate of $63.8 \%$. The consumption pattern showed that $84.9 \%$ had low risk / abstinent, II. I\% risky use, I.3\% harmful use and $2.6 \%$ possible dependence. There was an association between alcohol consumption with schooling, violence and discrimination.

Conclusion: High prevalence associated with factors such as violence, discrimination and education, requires greater attention to women who use alcohol.
\end{abstract}

Keywords: Women;Alcoholism; Primary Health Care; Risk factors.

I. Graduanda em enfermagem pela Universidade Federal do Piauí (UFPI), Curso de Enfermagem. Endereço: Campus Ministro Petrônio Portella, SG I I-Bloco Enfermagem-Ininga, Teresina - PI, 64049-550, Brasil.Email: eukaliarocha@yahoo.com.br

2. Professora doutora da Universidade Federal do Piauí, Programa de Pós-Graduação em Enfermagem. Endereço: Campus Ministro Petrônio Portella, SG I2 - Ininga, Teresina - PI, 64049-550, Brasil. Email: claudetefmonteiro@ufpi.edu.br

3. Professora doutora da Universidade Federal do Piauí. Endereço: Campus Ministro Petrônio Portella, SG I2 - Ininga, Teresina - PI, 64049-550, Brasil. Email: jaqueline-carvalho@uol.com.br

4. Doutora em enfermagem pela Universidade Federal do Piauí. Docente do curso de Enfermagem da Universidade Estadual do Piauí. Endereço: Rua Olavo Bilac, 2325 Centro, Teresina - PI, 640 I5-0I7, Brasil. Email: lorenaupveloso@gmail.com

5.Professor doutor da Universidade Federal do Piauí.Pós-Doutorado em Enfermagem pela Universidade Federal de São Carlos. Docente do Mestrado Profissional em Saúde da Família (UFPI). Endereço: Campus Ministro Petrônio Portella, SG I2 - Ininga, Teresina - PI, 64049-550, Brasil. Email: fernandoguedesjr@gmail.com

6. Cirurgião Dentista pelo Centro Universitário (Uninovafapi). Especialista em Saúde da Família e Ortodontia pela Uninovafapi. Cirurgião dentista da Estratégia Saúde da Família do município de Timon-MA. Endereço: Rua Vitorino Orthiges Fernandes, 6I23- Uruguai,Teresina - PI, 64073-505, Brasil. Email: thimon20@hotmail.com 


\section{INTRODUÇÃO}

O uso problemático de álcool é considerado um desafio social e econômico, tanto no cenário brasileiro quanto em outros países, ocupando o primeiro lugar mundialmente entre as substâncias psicoativas mais consumidas, figurando em ambos os sexos. Esse uso é considerado um problema de saúde pública, com repercussões na saúde física e mental dos usuários, nas suas relações familiares e gerando impacto socioeconômico(I).

As mulheres se tornam mais vulneráveis ao uso de bebida alcoólica quando comparadas aos homens, devida variações antropométricas, como a maior proporção de gordura corporal, além de diferenças no metabolismo hepático do álcool. Dessa forma, são mais susceptíveis aos estados de alcoolismo e possível dependência ${ }^{(2)}$.

Essa conduta vem com a prática do binge drinking. De acordo com o National Institute on Alcohol and Alcoholism (NIAA) a quantidade de álcool que determina esse padrão é de cinco ou mais doses em uma única ocasião por homens ou quatro ou mais por mulheres, desconsiderando a frequência de uso(3).

Quanto aos fatores que favorecem o consumo entre as mulheres está 0 início na adolescência, muitas vezes para vivenciar situações prazerosas, ou por serem muIheres que passaram por experiências de discriminação, vivência de perdas, agravos físicos e psicológicos que possuem relação direta com o uso abusivo de álcool(4).

Entre muitas situações ligadas ao álcool estão as violências, com destaque para a violência entre parceiros íntimos (VPI), na qual a mulher busca no álcool válvula de escape ou para esquecer situações traumáticas ${ }^{(5)}$. Esse envolvimento da mulher com o alcoolismo faz surgir o estigma e preconceito que dificulta sua busca por tratamento. Nesse sentido, ressalta-se a importância do fortalecimento da rede de atenção em saúde para acolher e dar suporte a população feminina em situação de dependência química, bem como ser uma rede capacitada para o contato com a usuária que busca o serviço.

A atenção primária em saúde é um dos principais meios de atendimento aos usuários de substâncias psicoativas como o álcool. Portanto, este nível de atenção em saúde deve possuir competências para prevenção do uso abusivo de álcool, implantando práticas educativas e terapêuticas, além de identificar precocemente usuários em situação de risco para uso de álcool(6).

Diante dessa problemática, o objetivo deste estudo foi analisar o padrão de consumo do álcool e fatores associados em mulheres.

\section{METODOLOGIA}

Estudo epidemiológico, transversal, realizado em Teresina/Piauí/Brasil. A pesquisa foi desenvolvida a partir do projeto "Impacto de intervenções para uso de dro- gas, sintomas depressivos, ansiosos e comportamento suicida na população adulta de Teresina" (Processo: 30557I/2017-6 - Chamada CNPq N ${ }^{\circ}$ I2/2017 - Bolsas de Produtividade em Pesquisa $-\mathrm{PQ}$ ), desenvolvido junto às 26 Equipes da Estratégia Saúde da Família.

A população fonte constou de 14.062 indivíduos, na faixa etária de 20 a 59 anos, cadastrados no sistema de informação e-SUS. Para o cálculo amostral levou-se em consideração uma prevalência presumida de uso de álcool entre a população adulta de $50 \%$, nível de confiança de $95 \%$ e erro máximo de $5 \%$, obtendo-se 374 indivíduos a serem entrevistadas. Por tratar-se somente de levantamento entre mulheres, da amostra pesquisada 305 eram mulheres. Como critério de inclusão foi considerado pesquisar somente as mulheres, totalizando 305 participantes. Como critérios de exclusão: participantes com problemas de audição e/ou diç̧ão, que dificultassem a transmissão clara das informações da pesquisa.

A coleta de dados foi realizada entre maio a julho de 2019, com os instrumentos: Escala Alcohol Use Desorders Identification Test (AUDIT) e um questionário estruturado e multidimensional, para aspectos sociodemográficos, condições de saúde e hábitos de vidas, elaborado especificamente para a pesquisa.

Para a análise de dados, realizou-se processo de dupla digitação, utilizando-se planilhas do Software Microsoft Excel e posteriormente exportados e analisados no software Statistical Package for the Social Science (SPSS), versão 20.0. Na caracterização da amostra utilizou-se estatísticas descritivas. Para verificar associação entre as variáveis qualitativas foi utilizado o teste qui-quadrado $\left(x^{2}\right)$. A força das associações entre as variáveis foi aferida pelo odds-ratio (OR) e intervalos de confiança (IC 95\%). Para o estudo das associações entre as variáveis quantitativas, utilizou-se o coeficiente de correlação de Spearman. Em todas as análises adotou-se nível de significância de 0,05.

As participantes assinaram um Termo de Consentimento Livre e Esclarecido (TCLE) e foram observados os demais aspectos éticos da Resolução $\mathrm{n}^{\circ}$. 466 de 12 de dezembro de 2012, do Conselho Nacional de Pesquisa. O estudo foi aprovado em Comitê de Ética em Pesquisa da Universidade Federal do Piauí (parecer $n^{\circ}$ 2.404.139).

\section{RESULTADOS}

Os dados mostraram que as mulheres estavam, em sua maioria, na faixa etária de 30 a 39 anos (36,7\%), com companheiro, em convívio mesmo lar $(63,9 \%)$, com mais de 10 anos de estudo (60\%), com filhos $(86,2 \%)$, da raça parda (62\%), classe econômica E (83,6\%), sem trabalho (59,3\%), com religião (92,1\%). Observou-se história de violência $(21,6 \%)$ e discriminação ( $17,4 \%)$.

Das 305 mulheres pesquisadas, I05 informou consu- 
mo de álcool nos últimos 12 meses, apresentando uma prevalência estimada em 34,4\%, com uso em "binge" de $63,8 \%(n=67)$.

Quanto ao padrão de consumo de álcool, $84,9 \%$ das participantes observou-se padrão de baixo risco ou abstinência, I I, I\% uso de risco, I,3\% uso nocivo e $2,6 \%$ possível dependência.

Entre os fatores associados ao consumo de álcool pelas mulheres está a escolaridade. Mulheres com até 10 anos de estudo tiveram proporções maiores de consumo de risco, nocivo e possível dependência quando comparadas às que informaram mais de 10 anos de estudo $(p=0,005)$. A violência e discriminação também mostrou associação com consumo de álcool. Mulheres que relataram ter sofrido algum tipo de violência $(p=0,003)$ ou discriminação em algum momento da vida $(p=0,003)$ são mais propensas ao consumo de álcool (Tabela I).
Na comparação de médias obtidas na aplicação do AUDIT, somente houve diferença de médias estatisticamente significativas para variáveis: estado civil $(p=0,025)$ e histórico de violência $(p=0,032)$ (Tabela 2$)$.

\section{DISCUSSÃO}

As variáveis sociodemográficas caracterizam o grupo de mulheres na faixa adulta jovem, do lar com companheiro, filhos, raça parda, com religião, com pouco mais de 10 anos de estudos, sem trabalho e pertencentes a menor classe econômica do país - E. Mostra também presença de violência e discriminação. Essa caracterização vem sendo apresentada por outros estudos, chamando atenção para grupos nessa faixa etária, com pouca escolaridade, relatos de violência e discriminação ${ }^{(7,8)}$.

A OMS tem alertado para o avanço do consumo do álcool em todo o mundo. Destaca que $43 \%$ da po-

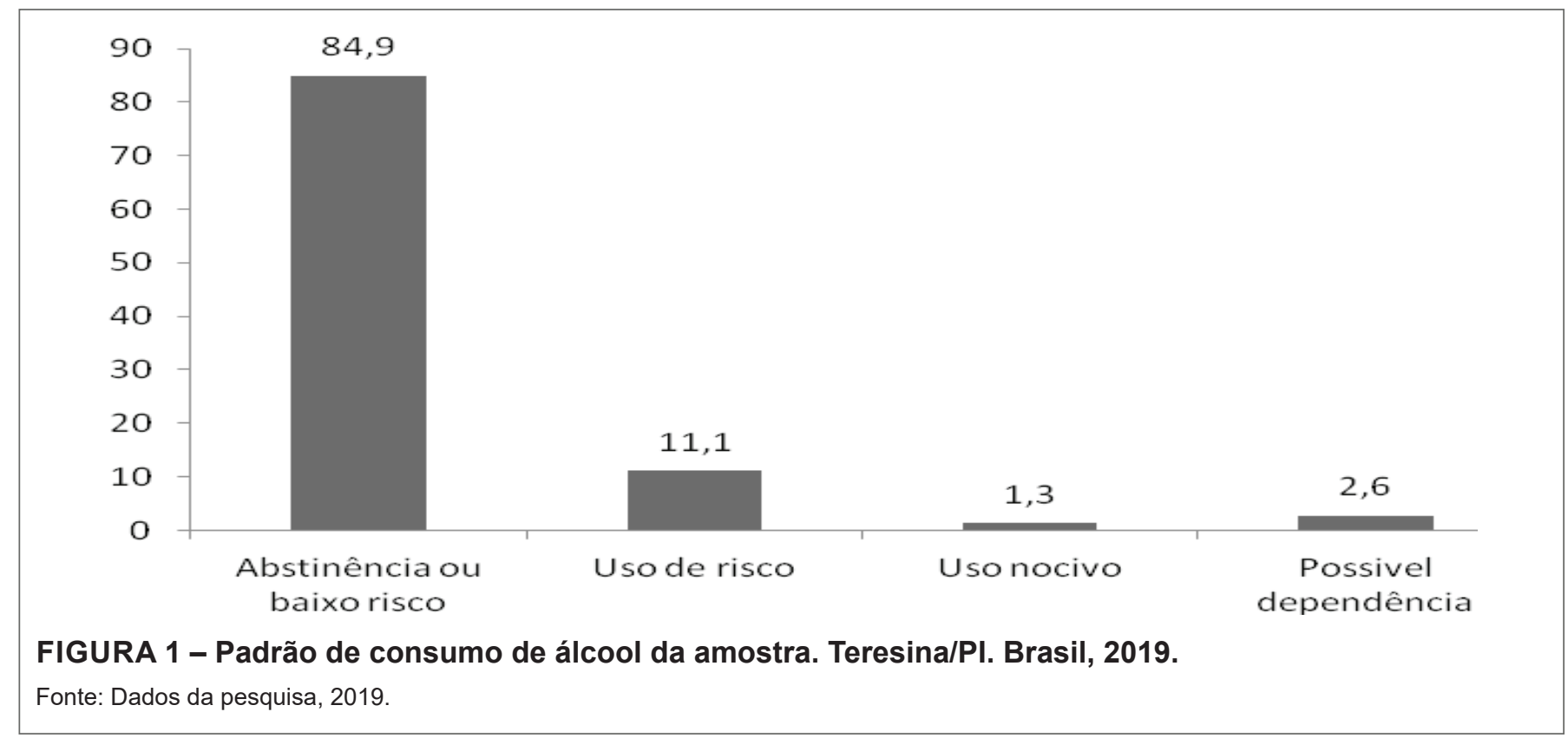

TABELA 1 - Fatores associados ao padrão de consumo de álcool entre mulheres. Teresina/PI. Brasil, 2019.

\begin{tabular}{|c|c|c|c|c|c|}
\hline \multirow[t]{2}{*}{ Variáveis } & \multicolumn{4}{|c|}{ Padrão de consumo de álcool } & \multirow[b]{2}{*}{$p^{*}$} \\
\hline & $\begin{array}{c}\text { Abstinência ou } \\
\text { baixo risco } \\
n(\%)\end{array}$ & $\begin{array}{c}\text { Uso de risco } \\
n(\%)\end{array}$ & $\begin{array}{c}\text { Uso nocivo } \\
n(\%)\end{array}$ & $\begin{array}{c}\text { Possível } \\
\text { dependência } \\
n(\%)\end{array}$ & \\
\hline \multicolumn{6}{|l|}{ Escolaridade } \\
\hline Até 10 anos de estudo & $87(34,9)$ & $19(63,3)$ & $4(100,0)$ & $6(75,0)$ & 0,005 \\
\hline Mais de 10 anos de estudo & $162(65,1)$ & $11(36,7)$ & $-(0,0)$ & $2(25,0)$ & \\
\hline \multicolumn{6}{|l|}{ História de violência } \\
\hline Sim & $48(18,5)$ & $13(38,2)$ & $1(25,0)$ & $4(50,0)$ & 0,003 \\
\hline Não & $211(81,5)$ & $21(61,8)$ & $3(75,0)$ & $4(50,0)$ & \\
\hline \multicolumn{6}{|l|}{ História de discriminação } \\
\hline Sim & & & & & 0,003 \\
\hline Não & $219(84,6)$ & $27(79,4)$ & $2(50,0)$ & $4(50,0)$ & \\
\hline
\end{tabular}

Fonte: Dados da pesquisa, 2019.

* Teste do Qui quadrado de Pearson 
pulação são bebedores atuais (consumiram nos últimos 12 meses), com média de 6,4 L de álcool puro. Chama também atenção para o fato de que embora a forte influência das politicas de prevenção tenha levado a uma diminuição no nível global de mortes e morbidade atribuíveis ao álcool, computa-se ainda um significativo ônus global de doenças atribuíveis ao seu consumo, cujo impacto para a morbidade, mortalidade e incapacidades está presente em todo o mundo. Para - Brasil, mostra associação do álcool com altos índices de cirrose hepática, acidentes de trânsito, câncer (entre homens e mulheres em 2016). Especificamente sobre os transtornos relacionados ao uso do álcool, estima-se que 4,2\% (6,9\% entre homens e I,6\% entre mulheres) dos brasileiros preenchem critérios para abuso ou dependência (9)

A prevalência do consumo de álcool nos últimos 12 meses levantada no estudo é considerada alta (34,4\%). Chama atenção o uso em "Binge" (63,8\%). Modo de beber considerado de risco à saúde devido ao elevado percentual de álcool ingerido em curto espaço de tempo - beber em binge é considerada a ingestão de 5 doses ou mais, no caso de homens, e 4 doses ou mais, no caso de mulheres, em uma mesma ocasião num intervalo de até 2 horas ${ }^{(2)}$. Tem sido observado um aumento significativo do uso em binge pelas muIheres nos últimos anos( ${ }^{(1)}$, fato que converge com os achados da presente pesquisa. Ressalta-se que o beber em binge favorece situações de violência, envolvimento em discussões, além dos riscos provenientes da intoxicação aguda por álcool. Considerando-se as mudanças observadas ao longo dos últimos anos no beber feminino, é importante destacar a necessidade de discussão da temática dentro das políticas em saúde voltadas para as mulheres.

O padrão de consumo levantado pelo instrumento AUDIT, nos últimos 12 meses aponta que, embora um percentual alto encontra-se no padrão de baixo risco ou abstinência, preocupa o padrão de consumo registrado por uso de risco, uso nocivo e possível dependência. As consequências do consumo abusivo de álcool por mulheres são mais rapidamente observadas do que nos homens. Doses mais baixas embriagam as mulheres mais rapidamente e o uso cotidiano avança para o alcoolismo crônico e com sérias complicações médicas.

Os dados mostram resultados também significativos quanto ao consumo de álcool e sua interface com a violência contra a mulher, mostrando associação significativa entre estas variáveis $(p=0,005)$. Entre os principais tipos de violência sofridos pela mulher está a violência física, destacando-se que o consumo pode ocorrer pela vítima após a situação de violência ${ }^{(10)}$. Abrangente estudo aponta que pessoas de ambos os sexos que foram expostas a situações de violência ainda na infância tem maior vulnerabilidade ao uso de álcool na vida adulta( ${ }^{(1)}$. Sugerindo assim uma possível relação de causalidade entre uso de álcool e violência. A discussão sobre esta relação é fundamental uma vez que a violência relacionada ao uso de álcool pode atingir diferentes grupos populacionais ${ }^{(12)}$.

A discriminação mostrou-se também associação significativa com consumo de álcool $(p=0,003)$. Estudos empíricos existentes sobre $o$ assunto trazem a hipótese de que experiências discriminatórias favorecem a adoção de comportamentos de risco à saúde, com destaque para o uso de substâncias psicoativas como o álcool. Essa associação sugere que pessoas vítimas de discriminação poderiam adotar o uso do álcool, como uma estratégia de enfrentamento e alívio do estresse resultante dessas experiências negativas ${ }^{(13)}$.

O uso problemático de álcool por mulheres tende a ser visto de maneira menos tolerante quando comparado aos homens, desta forma o estigma e preconceito podem tornar-se mais presentes na vida dessas mulheres ${ }^{(14)}$. Nesse sentido, a saúde mental da mulher e a possibilidade de seu envolvimento com álcool e outras drogas deve ser estudado e debatido pelos serviços de saúde, levando

TABELA 2 - Comparação de médias obtidas no escore AUDIT entre mulheres. Teresina/PI. Brasil, 2019

\begin{tabular}{|c|c|c|}
\hline Variáveis & $\begin{array}{l}\text { Escore } \\
\text { AUDIT }\end{array}$ & $P$ \\
\hline $\begin{array}{l}\text { Estado civil } \\
\text { Com companheiro, em convívio mesmo lar } \\
\text { Com companheiro, sem convívio } \\
\text { Sem companheiro, com casamento anterior } \\
\text { Sem companheiro }\end{array}$ & $\begin{array}{l}2,23 \\
5,17 \\
3,20 \\
3,39 \\
\end{array}$ & $0,025^{1}$ \\
\hline $\begin{array}{l}\text { História de violência } \\
\text { Sim } \\
\text { Não }\end{array}$ & $\begin{array}{l}4,69 \\
2,27\end{array}$ & $0,032^{2}$ \\
\hline
\end{tabular}

Fonte: Dados da pesquisa, 2019.

1 Teste de Kruskall Wallis, com nível de significância de 0,05

2 Teste de Mann Whitney, com nível de significância de 0,05 
a discussão à população de modo a tornar a problemática visível e passível de prevenção e tratamento.

Limitações observadas se referem ao método, pois estudos transversais apresentam limitações no tocante a vincular relações de causa e efeito, tendo em vista que avaliam uma realidade em determinado recorte de tempo.

\section{CONCLUSÃO}

O presente estudo permitiu identificar alta prevalência de consumo de álcool e elevado uso em binge, com padrão voltado ao consumo nocivo à saúde. Quanto aos fatores associados, chama à atenção a correlação encontrada entre o uso de álcool, violência e discriminação. 


\section{REFERÊNCIAS}

I. Munhoz TN, Santos IS, Nunes BP, Mola CL, Silva ICM, Matijasevich A. Tendências de consumo abusivo de álcool nas capitais brasileiras entre os anos de 2006 a 2013: análise das informações do VIGITEL. Cad. Saúde Pública [Internet] 2017 [acesso em 0I dez]; 33(7). Disponível em: http://dx. doi.org/10.1590/0102-311×00104516

2. Yue Y, Hong L, Guo L, Gao X, Deng J, Huang J et al. Gender differences in the association between cigarette smoking, alcohol consumption and depressive symptoms: a crosssectional study among Chinese adolescents. Sci Rep [Internet] 2015 [acesso em 0l dez]; 5:I7959. Disponível em: https://doi.org/10.1038/srep 17959

3. Wilsnack SC, Wilsnack RW, Kantor LW. Focus on: women and the costs of alcohol use. Alcohol Res [Internet]. 2013 Mar [acesso em 0I dez];35(2):219-28. Disponível em: https://www.ncbi.nlm.nih.gov/pmc/articles/PMC39087/3/

4. Caetano R, Madruga C, Pinsky I, Laranjeira R. Patrones de consumo de alcohol y problemas asociados en Brasil,Adicciones [Internet] 2013 [acesso em $05 \mathrm{dez}$ ] 25: 287-293. Disponível em: http://www.adicciones.es/index.php/adicciones/article/view/28

5. Martins AG, Nascimento ARA. Violência doméstica, álcool e outros fatores associados: uma análise bibliométrica. Arq. Bras. Psicol. [Internet]. 2017 [acesso em $18 \mathrm{dez}$ ]; 69(I): 107I2I. Disponível em: http://pepsic.bvsalud.org/scielo.php?script=sci_arttext\&pid=S 1809-526720 I 7000 I 00009\&lng=pt.

6. Jomar RT, Abreu AMM, Griep RH. Padrões de consumo de álcool e fatores associados entre adultos usuários de serviço de atenção básica do Rio de Janeiro, RJ, Brasil. Ciênc. Saúde coletiva [Internet]. 2014 Jan [acesso em $02 \mathrm{dez}$ ]; 19(I): 27-38. Disponível em: http://dx.doi.org//0.1590//4/381232014191.2009 .

7. Massignam FM, Bastos JLD, Nedel FB. Discriminação e saúde: um problema de acesso. Epidemiol. Serv. Saúde [Internet] 2015 [acesso em $18 \mathrm{dez}$ ]; 24(3):54I-544. Disponível em: http://scielo.iec.gov.br/scielo.php?script=sci_arttext\&pid=SI 679-497420 I 5000300020\&Ing=pt.

8. Silva MGB, Lyra TM, Diniz GT. O padrão de consumo de álcool entre as usuárias das Unidades de Saúde da Família no município do Recife (PE). Saúde debate [Internet]. 2019 [acesso em 03 nov 2019] Disponível em: http://dx.doi. org/10.1590/0103-1104201912214.

9. Organização Mundial da Saúde (OMS). Relatório Global sobre Álcool e Saúde - 2018. Genebra, Suiça

10. Zanoti-Jeronymo DV, Zaleski MJB, Pinsky I, Caetano R, Figlie NB, Laranjeira, RR. Violência na infância, exposição a violência parental e abuso e/ou dependência de álcool na idade adulta*. SMAD. Revista eletrônica saúde mental álcool e drogas [Internet]. 2019 [acesso em 12 nov 2019]; I5(I): 40-49. Disponível em: https://dx.doi.org/l0.I 1606/ issn. 1806-6976.smad.2019.000390

II. Martins AG, Nascimento ARA. Violência doméstica, álcool e outros fatores associados: uma análise bibliométrica. Arq. bras. psicol. [Internet]. 2017 [acesso em 12 nov 2019]; 69(I):I07-121. Disponível em: http:// pepsic.bvsalud.org/scielo.php?script $=$ sci_arttext\&pi$d=S|809-526720| 7000100009 \& \mid n g=p t$.

12. Tondowski CS, Feijó MR, Silva EA, Gebara CFP, Sanchez ZM, Noto AR. Intergenerational patterns of family violence related to alcohol abuse: a genogram-based study. Psicologia: Reflexão e Crítica [Internet] 2014 [acesso em 12 nov 2019]; 27(4): 806-8|4. Disponível em: https://dx.doi. org/I0.1590/|678-7|53.20142742 I

13. Matsumoto DS. Mulheres e álcool: uma questão de gênero. Serv. Soc. e Saúde [Internet]. 2015 [acesso em 12 nov 2019]; I2(2):237-5. Disponível em: https://periodicos.sbu. unicamp.br/ojs/index.php/sss/article/view/8639497

14. Lima-Rodríguez, JS, Guerra-Martín, MD, Domínguez-Sánchez, I, Lima-Serrano, M. Resposta da pessoa doente alcoólatra frente à sua doença: perspectivas de pacientes e familiares. Revista Latino-Americana de Enfermagem [Internet] 2015 [acesso em 12 nov 2019];23(6):II65II72. Disponível em: https://www.redalyc.org/articulo. oa?id=28 | 4428 | 1023

Recebido: 2020-03-17

Aceito: 2020-03-05 